Determining Efficacy of the Combined Application of Electrical Stimulated Antagonist Contraction During Walking with Sensory TENS for Increasing Strength and Decreasing Pain in Women with Frequent Knee Symptoms

NCT02730598

December 8, 2016 
Title

Randomized, controlled, pilot study

Determining Efficacy of the Combined Application of Electrical Stimulated Antagonist Contraction During Walking with Sensory TENS for Increasing Strength and Decreasing Pain in Women with Frequent Knee Symptoms

\section{Background \& Rationale}

Osteoarthritis of the knee (KOA) is the most common cause of disability not only in the United States but also in Japan. KOA is associated with pain, quadriceps weakness, swelling, instability, decline of range of motion, physical function, and quality of life (QOL) (1). In particular, quadriceps weakness may contribute to incident symptomatic and progressive disease $(2,3)$, cause functional limitations and disability (4) and increase the risk of mortality (5). Both aerobic exercise and resistance exercise are recommended for the treatment of people with knee osteoarthritis (KOA) (6). However, exercise at a moderate or severe intensity is often a problem for people with knee pain or a history of knee injury. Neuromuscular electrical stimulation (NMES) is widely used as a method to increase muscle strength and improve physical function even at a low-moderate exercise intensity (7). However, NMES effect may prove to be insufficient because the exercise intensity is determined by the electrical stimulation endurance level of the user (7). On the other hand, transcutaneous electrical stimulation, so-called transcutaneous electrical nerve stimulation (TENS), is effective for pain relief (8). Knee pain independently reduced quadriceps strength and activation (9). TENS restores inhibited quadriceps motor function (central and muscle activation) through pain relief (10). Moreover the combined application of electrical stimulation (ES) and volitional contractions (VC) is said to be more effective than ES or VC alone (11). Therefore, a hybrid training system (HTS) that resists the motion of a volitionally contracting agonist muscle using the force generated by its electrically stimulated antagonist (NMES) was developed as a way to combine the application of electrical stimulation and voluntary contraction (12). HTS is a method that eliminates the disadvantages of both volitional exercise and NMES (13). It has been reported that HTS is a new training technique that can increase both muscular strength and muscle mass $(12,13,14,15)$. Recently, HTS is showing promise as a countermeasure for the musculoskeletal disuse of astronauts because the HTS technique can generate exercise resistance within the body even if there is no gravity (1). In addition, we can utilize HTS during many different types of exercise (e.g. knee extension exercise, squat and hip flexion, walking exercise, and cycling exercise) $(17,18)$. It seems to be more effective for improvement of muscle strength and physical function to combine HTS with easy exercise (e.g. knee extension, walking, and squats) for KOA patients.

The main purpose of exercise therapy for KOA is not only muscular strength improvement (the quadriceps femoris muscle in particular) and but also pain relief $(19,20)$. Muscular strength improvement protects joints and relieves nociceptive stimulation. However, exercise sometimes increases pain. In addition to the pathological change in articular structures, changes in central pain processing or central sensitization appear to be involved in KOA pain (19). Murphy et al. reported that $36 \%$ of a heterogenous sample of patients with hip and KOA demonstrated evidence of central sensitization (22). This finding has been incompletely characterized, but it is necessary to consider central sensitization in the treatment of patients with KOA. From a theoretical perspective, exercise has the potential to treat the process of central sensitization: e.g. exercise activates brain-orchestrated endogenous analgesia (23). An initial bout of high intensity eccentric exercise induces central sensitization, but a repeated round of exercise facilitates inherent protective spinal mechanisms (repeated bout effect) (24). Moreover, ipsilateral resistance exercise may possibly prevent the central sensitization (25). Therefore, a time-contingent approach which implies that the patient does not cease exercise bouts once local pain severity increases is recommended (22). A few reports about the dysfunctional endogenous analgesia for patients with musculoskeletal pain response to aerobic exercise were shown, but neither type of aerobic exercise was able to activate endogenous analgesia (24). At present, there is no report of an exercise method that is effective in central sensitization pain patients. Brain-derived neurotrophic factor (BDNF) belongs to the neurotrophic family of growth factors. The loss of BDNF usually leads to neurodegeneration in these motor centers and eventually results in several severe motor diseases, such as amyotrophic lateral sclerosis, spinocerebellar ataxias, Parkinson's disease, Huntington's disease, as well as vestibular syndrome. These neurotrophic factors (e.g., 
decreasing brain-derived neurotrophic factor) are promising new avenues for diminishing hyperexcitability of the CNS in central sensitization pain patients (2). Da Graca-Tarrago et al. showed that a 30-minute electrical intramuscular stimulation in osteoarthritis decreased pain, increased the local pain pressure threshold (PPT), and decreased BDNF (27). Gajewska-Wozniak et al. reported that lowthreshold electrical stimulation of peripheral nerves to stimulate Ia afferent fibers (proprioceptive signaling) might affect the expression of BDNF in rats (28). HTS is an exercise technique that uses electrically eccentric muscle contraction. Yamaguchi et al. showed that the soleus H-reflex increased after one HTS adversely in conventional resistance exercise (29). This seems to indicate that HTS serves to activate Ia fibers. HTS may affect central sensitization and relieve pain in KOA patients.

\begin{abstract}
Aim:
In this pilot study, we will use walking with TENS as conventional exercise.

Randomized controlled trial will be conducted to compare the effect of walking augmented by HTS with walking without HTS. We will evaluate the relative advantages of training that combines HTS with conventional walking exercise on the improvement of muscle strength, physical function, and pain relief in obese women with frequent knee symptoms.
\end{abstract}

The primary outcome will be change in knee extensor strength.

The secondary outcome will be change knee pain.

Study Hypotheses: Compared with walking with sensory TENS, walking with HTS will: \#1 increase quadriceps muscle strength.

\#2 decrease knee pain.

Exploratory Hypotheses:

\#3 improve physical function.

\#4 increase PPT (improve central sensitization).

\#5 improve self-reported quality of life.

Study period: 2016/2/1 2016/08/31

Study location: Study procedures will take place in the CTSU ( $3^{\text {rd }}$ floor) or Kirmayer Fitness Center.

\title{
Study Population:
}

Inclusion Criteria

There will be a total of 28 participants recruited to participate in this study.

1) Female

2) Age 40-70 years

3) Knee symptoms (pain, aching, or stiffness) on most of the last 30 days (categorically defined)

4) Body Mass Index (BMI) $30-45 \mathrm{~kg} / \mathrm{m}^{2}$

\section{Exclusion Criteria}

1) Resistance training at any time in the last 3 months prior to the study

2) Knee replacement

3) Lower limb amputation

4) Lower limb surgery in the last 6 months that affects walking ability or ability to exercise

5) Back or hip problems that affect walking ability or ability to exercise

6) Unable to walk without a cane or walker

7) Inflammatory joint or muscle disease such as rheumatoid or psoriatic arthritis or polymyalgia rheumatica

8) Multiple sclerosis or other neurodegenerative disorder

9) Known neuropathy

10) Self-report of Diabetes 
11) Currently being treated for cancer or having untreated cancer

12) Terminal illness (cannot be cured or adequately treated and there is a reasonable expectation of death in the near future)

13) Peripheral Vascular Disease

14) History of myocardial infarction or stroke in the last year

15) Chest pain during exercise or at rest

16) Use of supplemental oxygen

17) Inability to follow protocol (e.g. lack of ability to attend visits or understand instructions)

19) Staff concern for participant health (such as history of dizziness/faintness or current restrictions on activity)

20) Unable to attend more than 2 days within any 1 week or unable to attend 4 or more sessions during the study

21) Implanted cardiac pacemaker, spinal cord stimulator, baclofen or morphine pump or other implanted electrical device.

22) Dermatitis or skin sensitivity to tape used in the study.

23) Pregnancy

\section{Sample Size Estimate}

The primary analysis will be the comparative assessment of the effect of HTS during walking on strength vs. walking with sensory TENS using RCT design. A sample size was estimated based upon prior data collected for the clinically significant difference in isokinetic quadriceps torque (30) and appropriate standard deviations within and between groups. At a significance level of 0.05 (adjusted for two outcomes: strength and pain), a standard deviation in the quadriceps strength response variable of $12.2 \mathrm{Nm}$, and a power of 0.80 , to detect an inter-group difference in means of $14.6 \mathrm{Nm}$ would require a minimum of 24 subjects for this two-treatment parallel-design study. To account for up to $15 \%$ drop-out, 14 subjects will be recruited into each of the 2 groups.

\section{Interventions:}

Training will occur for 30 minutes per session, 2 times a week for 12 weeks (total of 24 sessions). Each session will be separated by an interval of at least 48 hours. The subjects perform the walking exercise with HTS or with sensory TENS. The subjects will be instructed to avoid excessive exercise outside of the study participation to avoid over-fatigue during the study period. During training, an exercise specialist will be continuously present to provide guidance and monitoring in order to ensure that training is performed safely and properly. Each training session will begin and end with 5 minutes of supervised warm-up and cool-down. Prior to and following exercise training, subjects will be asked whether they are feeling light-headed, faint, headache or sick and will not be trained or discharged if they are feeling ill.

Hybrid Training System

On the anterior thigh, electrodes will be placed over the quadriceps, while on the posterior thigh, electrodes (Sekisui Plastics Co., Tokyo, Japan) will be placed over the hamstrings. Size of the electrodes is $15 \mathrm{~cm} \times 6 \mathrm{~cm}$ for the quadriceps and $11 \mathrm{~cm} \times 6 \mathrm{~cm}$ for the hamstrings, and they are coated with an oxidation-resistant silver-carbon compound and low impedance gel coating. 


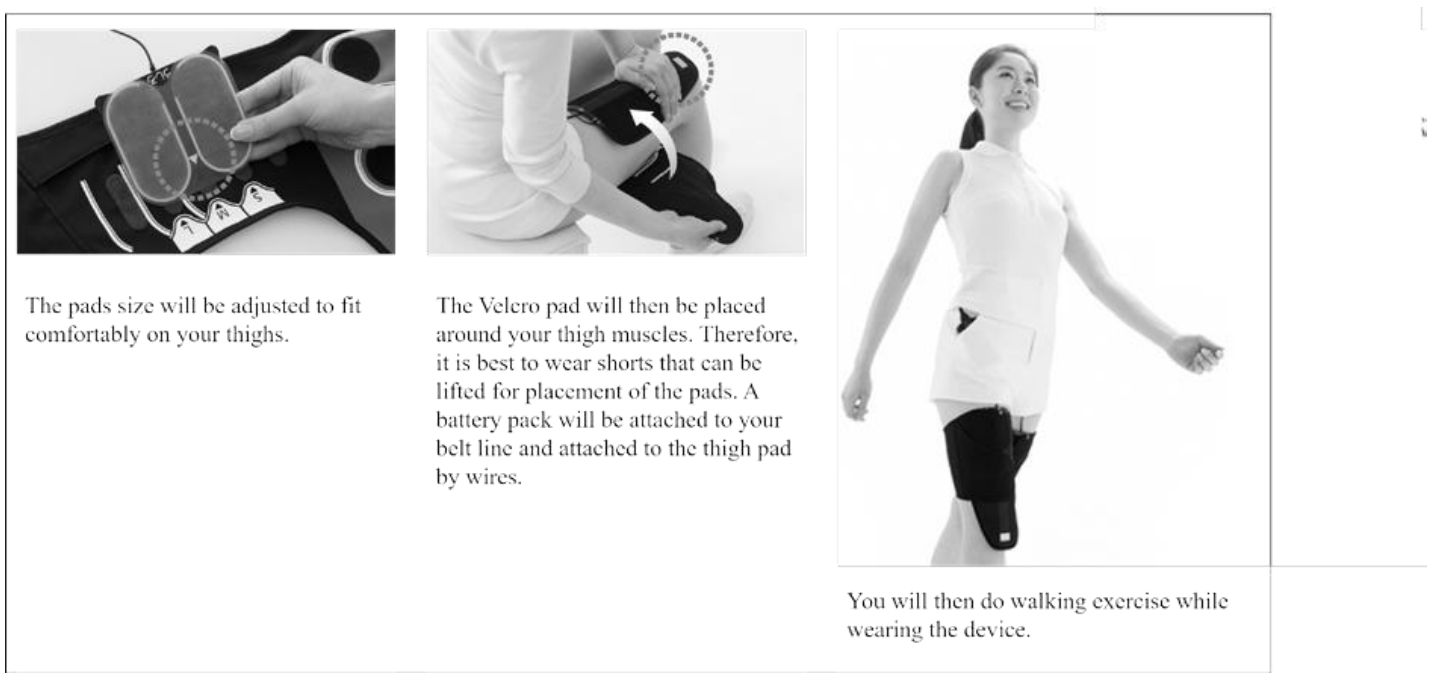

Electrical stimulation parameters will be based on a standard Russian waveform in which a $5,000 \mathrm{~Hz}$ carrier frequency is modulated at $40 \mathrm{~Hz}(2.4 \mathrm{~ms}$ on, $22.6 \mathrm{~ms}$ off $)$ to deliver a rectangular voltage biphasic pulse. Acceleration sensors as a joint motion sensor (EWTS9PD, Home Appliances Development Center Corporate Engineering Division, Appliances Company Panasonic Corporation 2-3-1-2 Nojihigashi,Kusatsu City, Shiga, Japan) is placed on the front of each leg $88 \mathrm{~mm}$ above the patellar edge. It analyzes the algorithm of each exercise pattern, and stimulates the antagonist of the motion of each bilateral knee joint during exercise.

Electrical stimulation intensity will be set to approximately $50-60 \%$ of $1 \mathrm{RM}$ based on the subject's tolerance. This intensity successfully improves muscle strength and mass without causing pain or numbness (14). Because the subject's tolerance gradually increases, electrical stimulation intensity is reset every 2 weeks.

Safety

The electrical stimulation device has a stimulus pattern with interlock and limiter for safety. Therefore, the effective current is interlocked at $11-12 \mathrm{~mA}(1 \mathrm{k} \Omega)$, and the peak voltage and current is limited to under $80 \mathrm{~V}$ and $90 \mathrm{~mA}$. These specifications meet a safety level of the Japanese Industrial Standards.

\section{Sensory TENS}

The electrical stimulation intensity will be set under the muscle contraction threshold (but at a level at which the subject can perceive as sensory TENS). Electrical stimulation parameters (i.e. waveform and pulse duration) will be the same of HTS, while the amplitude will be lower. The subject will be stimulated using the same device as for HTS.

Walking exercise with HTS or with sensory TENS

The walking training will be conducted for 30 minutes. The subject will walk at a comfortable pace. During walking, both lower extremities will be stimulated using the HTS device in response to the gait phase of each foot. Electrical stimulation of the quadriceps starts gradually from just before heel contact and stops with heel off (only during stance phase). Conversely, electrical stimulation of the hamstring starts gradually from just before heel off and ends with heel contact (swing phase). If the subject is tired and cannot continue walking, the subject will be instructed to take breaks as needed and then to continue walking once able. 


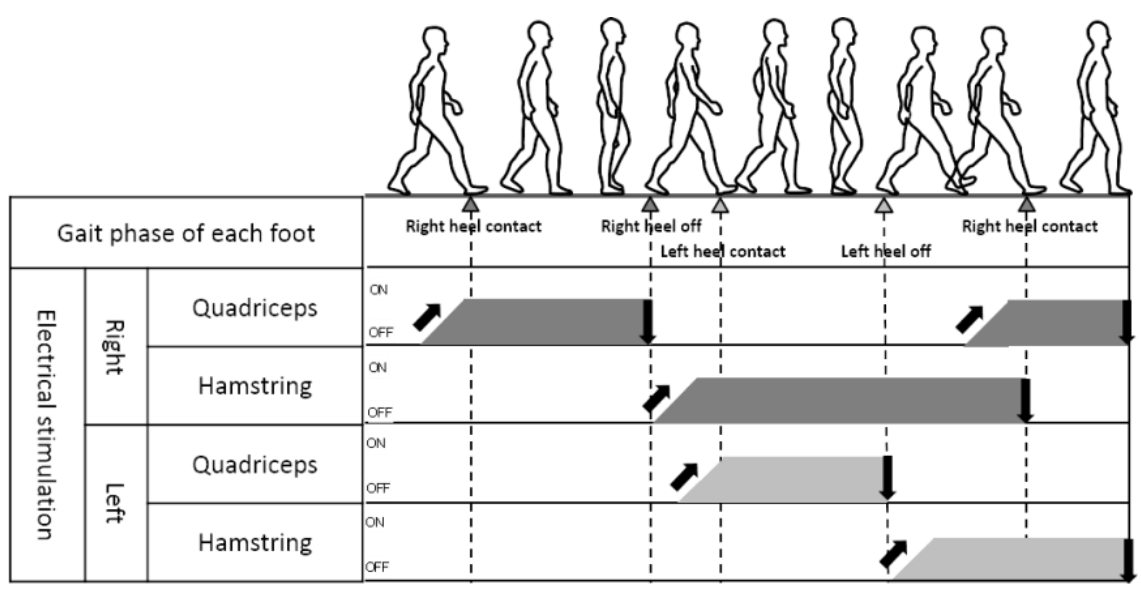

Outcome Assessments

Schedule of Measures

\section{Statistical Methods:}

Subject demographics will be described using means \pm SD for continuous variables and percentages for categorical variables.

We will use a one-sample t-test to assess changes from the baseline in each group (with/without HTS).

We will use repeated measures ANOVA (within factor: time and between groups).

\section{How Future Studies Will Differ:}

Future studies will be randomized controlled trials, with stricter inclusion and exclusion criteria. Future studies will also have a sufficient sample size to compare quantitative outcome measures (e.g. strength, muscle size, trajectory of knee pain) between intervention and control participants. Future studies will also have a measurement method that can evaluate a central nerve function more.

Description of the enrollment and consent process for participants:

Information about the study will be posted in local businesses, local events (such as health fairs), on the KUMC research website, in newsletters and other publications, and via a KU all-user email notice (staff, faculty, and alumni). If interested in the study, the potential participant will contact study staff either in person (if at a health fair for example) or by phone to discuss their participation and eligibility for the study. If eligible and interested in the study, the potential participant will be scheduled for an assessment visit. If time permits, a letter and a copy of the consent form will be mailed to the potential participant, which will include directions to the appointment and what to expect at the appointment. At the beginning of the assessment visit, the potential participant will participate in an informed consent process, culminating in signing the informed consent document. A copy of the signed informed consent document will be given to the participant.

\section{Questionnaires, surveys, written assessments that will be used to obtain data directly from} participants in this study:

- Baseline visit data collection form

- Phone screen

- Knee and Osteoarthritis Outcome Score (KOOS) - at baseline visit

- Visual Analog Scale (VAS) for pain at each visit

\section{Procedures following the consent process:}

Baseline Assessment: (135 min)

Participants who have met initial eligibility criteria by the phone screen will attend a 55-minute baseline visit with the study coordinator to undergo the following:

1) Informed Consent Process: At the beginning of this baseline assessment visit, potential 
participants will undergo an informed consent process. (30min)

2) Confirm eligibility: 1) Vital Signs: The participant will have blood pressure checked to ensure it is less than 180/100. If not within this range, the participant will not be eligible to take part in the study. 2) Height and body weight: BMI will be calculated from them. (5min)

3) Knee Symptoms and Function will be assessed with the Knee Osteoarthritis Outcome Score (KOOS). The KOOS is a 42-item self-administered questionnaire that covers 5 patient-relevant dimensions: Pain, Other Disease-Specific Symptoms, activities of daily living (ADL), Sport and Recreation, and Knee-related Quality of Life (QOL). This instrument is reliable and responsive in people with knee OA, and sensitive to changes. (20min)

4) Pain scale by a visual analog scale (VAS): Knee pain will be evaluated using a VAS of $10 \mathrm{~cm}$ from no pain to the worst imaginable pain. Participants will be asked VASs of the past one week. And participants will be asked once during the following physical performance test (walks, stands from a chair, and climbs stairs).

5) Measurement time: The time (seconds) is evaluated during 5 times standing up from a chair, 20m straight walking on the floor at their fastest comfortable pace, and stair climb. We will conduct each test two times. Second trial will be performed 2 minutes after first trial. (20min)

6) Isokinetic Strength Determination: Participants will be familiarized with strength testing equipment and counseled on proper lifting technique. They will undergo testing to determine their peak isokinetic knee extensor torque, using an isokinetic dynamometer. Participants will be seated on a chair with a hip joint angle of $85^{\circ}$. The center of rotation of the knee joint will be visually aligned with the axis of rotation of the dynamometer. The right shank will be firmly attached to the lever arm of the dynamometer with a padded strap. After correction for gravitational torque and a warm up with sub-maximal contractions, participants will be instructed to perform 4 maximal isokinetic knee extensions and flexions at $60^{\circ} / \mathrm{sec}$ through their maximal range of motion between $90^{\circ}$ of knee flexion and extended straight. These testing procedures will then be repeated for the left side. (40min)

7) Pressure pain threshold (PPT): PPT will be measured using a hand-held pressure algometer (FPIX compact digital force gauge, Wagner Instruments, USA). The probe (size $1 \mathrm{~cm}^{2}$ ) will be applied perpendicular to the skin until the participant first reports that the sensation of pressure has changed to pain. PPTs will be measured three times at each of the following sites: (1) bilateral styloid process of radius and (2) center of the patella. The average of the three measurements at each site will be used in the analysis. (10min)

8) Temporal summation: Temporal summation will be defined as being present when, after touching the skin over the bilateral 1) styloid process of radius and 2) patella with a $60 \mathrm{~g}$ monofilament repeatedly at $1 \mathrm{~Hz}$ for $30 \mathrm{~s}$, the subjects will report new or increasing pain. (10min)

Immediately before and after the exercise session Assessment Visit 1, 2, 3, 12, and 24 (20 min):

1) Pressure pain threshold (PPT): PPT will be measured using a hand-held pressure algometer (FPIX compact digital force gauge, Wagner Instruments, USA). The probe (size $1 \mathrm{~cm}^{2}$ ) will be applied perpendicular to the skin until the participant first reports that the sensation of pressure has changed to pain. PPTs will be measured three times at each of the following sites: (1) bilateral styloid process of radius and (2) center of the patella. The average of the three measurements at each site will be used in the analysis. (10min)

2) Temporal summation: Temporal summation will be defined as being present when, after touching the skin over the bilateral 1) styloid process of radius and 2) patella with a $60 \mathrm{~g}$ monofilament repeatedly at $1 \mathrm{~Hz}$ for $30 \mathrm{~s}$, the subjects will report new or increasing pain. (10min)

3) Pain scale by a visual analog scale (VAS): Knee pain is evaluated using a VAS of $10 \mathrm{~cm}$ from no pain to the worst possible pain. VASs will be evaluated before and after each exercise session and during each exercise session.

(This is included in exercise session)

Post-training period Assessment Visit 25 (120 min):

1) Knee Symptoms and Function will be assessed with the Knee Osteoarthritis Outcome Score (KOOS). The KOOS is a 42-item self-administered questionnaire that covers 5 patient-relevant dimensions: Pain, Other Disease-Specific Symptoms, activities of daily living (ADL), Sport and Recreation, and Knee-related Quality of Life (QOL). This instrument is reliable and responsive in people with knee OA, and sensitive to changes. (20min) 
2) Pain by a visual analog scale (VAS): Knee pain will be evaluated using a VAS will 0 representing no pain and 10 representing the worst possible pain. Participants will be asked VASs of the past one week. And participants will be asked once during the following physical performance test (walks, stands from a chair, and climbs stairs). (5min) (This is included in the following measurement).

3) Measurement time: The time (seconds) will be evaluated during 5 times standing up from a chair, $20 \mathrm{~m}$ straight walking on the floor, and stair climb. We will conduct each two times of test. Second trial will be performed 2 minutes after first trial. (20min)

4) Isokinetic Strength Determination: Participants will be familiarized with strength testing equipment and counseled on proper lifting technique. They will undergo testing to determine their peak isokinetic knee extensor torque, using an isokinetic dynamometer. Participants will be seated on a chair with a hip joint angle of $85^{\circ}$. The center of rotation of the knee joint will be visually aligned with the axis of the lever arm of the dynamometer. The right shank will be firmly attached to the lever arm of the dynamometer with a padded strap. After correction for gravitational torque and a warm up with sub-maximal contractions, participants will be instructed to perform 4 maximal isokinetic knee extensions and flexions at $60^{\circ} / \mathrm{sec}$ through their maximal range of motion between $90^{\circ}$ of knee flexion and extended straight. These testing procedures will then be repeated for the left side. (60min)

4) Pressure pain threshold (PPT): PPT will be measured using a hand-held pressure algometer (FPIX compact digital force gauge, Wagner Instruments, USA). The probe $\left(\right.$ size $\left.1 \mathrm{~cm}^{2}\right)$ will be applied perpendicular to the skin until the participant first reports that the sensation of pressure has changed to pain. PPTs will be measured three times at each of the following sites: (1) bilateral styloid process of radius and (2) center of the patella. The average of the three measurements at each site will be used in the analysis. (10min)

5) Temporal summation: Temporal summation will be defined as being present when, after touching the skin over the bilateral 1) styloid process of radius and 2) patella with a $60 \mathrm{~g}$ monofilament repeatedly at $1 \mathrm{~Hz}$ for $30 \mathrm{~s}$, the subjects will report new or increasing pain. (10min)

\section{Compensation for participation in Study}

Participants will be provided parking at no cost for appointments.

Participants who are eligible and participate in the study will receive $\$ 25$ for completing the initial assessment and $\$ 75$ after completing the final assessment visit. The amount of compensation will be prorated based on the duration of participation such that everyone who completes the initial assessments will receive $\$ 25$ plus $\$ 75$ divided by the number of exercise sessions of study participation (for example, $12 / 24$ sessions would receive $\$ 25+\$ 37.5$, whereas $24 / 24$ sessions would receive $\$ 25+\$ 75$ ). Payments will be disbursed via the ClinCard system.

\section{What are the risks to participants?}

Risks and discomforts include muscle soreness associated with exercise session or strength testing. This is a natural, expected phenomenon that occurs with exercise, particularly when a person has been inactive for a period of time. This type of soreness is expected to be transient. Also, there is possible discomfort associated with electrical stimulation. Other risks and discomforts include the possibility of muscle strain associated with improper technique or attempting to work with a resistance that is not appropriate. As with any physical activity, there is a risk of a cardiovascular event, such as abnormal blood pressure, fainting, irregular heartbeat, or cardiac ischemia and heart attack. There is the possibility of uncovering an existing problem during the study. There is also a possibility of contact dermatitis to the surface electrode gel.

\section{What will be done to minimize the risks?}

The KOOS questionnaire entails little risk. Information will be kept as confidential as possible through the use of code numbers on all forms and in all data analysis programs. No individual analyses will be performed using the data. Paper forms will be stored at the clinic office site in filing cabinets, which 
are locked, and offices are locked outside of work hours.

Risk of cardiovascular events is minimized through the use of screening questions. All exercise training staff are trained in CPR. Risks of muscular discomfort and strains are minimized through the use of warm-ups, stretching exercises during exercise sessions, and cool-downs. Qualified personnel, trained in the principles of exercise physiology, will supervise all training sessions, to ensure that proper form and technique is maintained, and that study participants, although expected to exercise at a challenging intensity, are not taking on more than they can safely handle.

All participant information is kept confidential, primarily through restricted access of participants' data and records. Only the research staff members have access to the study participants' data and records. Records are kept in a locked office. When participants are randomized, they are assigned a number to identify them in the database. Our research does not involve sensitive matters such as sexual or criminal behavior, and therefore, we have not deemed it necessary to obtain a Certificate of Confidentiality. Participant information will not be disclosed outside of the University of Iowa, except when medically necessary, i.e. if a medical condition is discovered during the course of the study, we will disclose that information to the study participant's primary care physician with his/her permission.

The examinations of muscular performance essentially mimic activities of daily living, but are performed on machines. Participants are asked if they may be unable to perform an activity such as climbing stairs, and if they feel they may be unable, that is recorded and the examination is not done. Participants will be verbally and visually monitored continuously during the intervention visits and will be provided with rest breaks and water whenever needed.

The surface electrodes are hypoallergenic.

\section{Stopping Criteria:}

Human subjects can elect to discontinue at any time. The exercises that will be completed are unloaded (other than the weight of their leg) and low intensity. If participants either appear to research team members to be or state that they 1) feel ill, 2) have increasing pain, or 3) are unable to complete the protocol, their participation will be discontinued. If they are found to have systolic/diastolic blood pressure exceeding 180/100, then they will not participate in the exercise.

\section{What are the direct benefits to the participants?}

No direct benefits can be guaranteed, but participants may experience benefits of various exercises and attention for the KOA. That is, we expect to see improvements in muscular function with intervention, and therefore activities of physical function.

What are the potential benefits to society in terms of knowledge to be gained as a result of this project?

The results will inform more specific rehabilitation interventions to increase lower limb strength that could potentially reduce knee pain. The ability to offer effective rehabilitation will contribute to independent mobility, enhanced health and health-related quality of life and inform public health and individual clinical decisions.

\section{What will be done to protect the privacy interests of the participants?}

To protect the privacy interests of the participants, all of the information collected during the study is collected using the same attention to patient privacy used during clinical care activities (e.g. all data is collected in private rooms and data is only available to individuals directly collecting the data). Paper forms with participant information and data will be stored in a locked file drawer, in a locked office, on the $3^{\text {rd }}$ floor of the CTSU.

\section{References:}

1. Muraki S, Akune T, Oka H, En-yo Y, Yoshida M, Saika A, Suzuki T, Yoshida H, Ishibashi H, Tokimura F, Yamamoto S, Nakamura K, Kawaguchi H, Yoshimura N. Association of radiographic and symptomatic knee osteoarthritis with health-related quality of life in a population-based cohort 
study in Japan: the ROAD study. Osteoarthritis Cartilage. 18(9):1227-34, 2010.

2. Segal NA, Glass NA, Felson DT, Hurley M, Yang M, Nevitt M, Lewis CE, Torner JC. The Effect of Quadriceps Strength and Proprioception on Risk for Knee Osteoarthritis. Med Sci Sports Exerc. Nov 42(11):2081-8, 2010.

3. Segal NA, Glass NA, Torner J, Yang M, Felson DT, Sharma L, Nevitt M, Lewis CE. Quadriceps weakness predicts risk for knee joint space narrowing in women in the MOST cohort. Osteoarthritis Cartilage Jun 18(6):769-75, 2010.

4. Van Dijk GM, Dekker J, Veenhof C, van den Ende CHM. Course of functional status and pain in osteoarthritis of the hip or knee: a systematic review of the literature. Arthritis Rheum. 55(12):779$85,2006$.

5. Nüesch E, Dieppe P, Reichenbach S, Williams S, Iff S, Jüni P. All cause and disease specific mortality in patients with knee or hip osteoarthritis: population based cohort study. BMJ. 8; 342:d1165, 2011.

6. Linda S Pescatello, Ross Arena, Deborah Riebe, Paul D Thompson. ACSM's Guidelines for Exercise Testing and Prescription by American College of Sports Medicine., 9nd ed., Wolters Kluwer/Lippincott Williams \& Wilkins, Philadelphia, PA. 2014

7. Giggins O, Fullen B, Coughlan G. Neuromuscular electrical stimulation in the treatment of knee osteoarthritis: a systematic review and meta-analysis. Clin Rehabil. 26(10):867-81, 2012.

8. Rutjes AW, Nüesch E, Sterchi R, Kalichman L, Hendriks E, Osiri M, Brosseau L, Reichenbach S, Jüni P. Transcutaneous electrostimulation for osteoarthritis of the knee. Cochrane Database Syst Rev. 2009 Oct 7;(4)

9. Park J, Hopkins JT.

Induced anterior knee pain immediately reduces involuntary and voluntary quadriceps activation.

Clin J Sport Med. 2013 Jan;23(1):19-24.

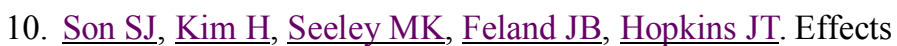

of transcutaneous electrical nerve stimulation on quadriceps function in individuals with experimentalknee pain. Scand J Med Sci Sports. 2015 Sep 8.

11. Paillard T. Combined application of neuromuscular electrical stimulation and voluntary muscular contractions. Sports Med. 38(2):161-177, 2008.

12. Yanagi T, Shiba N, Maeda T, Iwasa K, Umezu Y, Tagawa Y, Matsuo S, Nagata K, Yamamoto T, Basford JR. Agonist contractions against electrically stimulated antagonists. Arch Phys Med Rehabil 84(6):843-8, 2003.

13. Matsuse H, Shiba N, Umezu Y, Nago T, Tagawa Y, Kakuma T, Nagata K, Basford JR. Muscle training by means of combined electrical stimulation and volitional contraction. Aviat. Space Environ. Med. 77(6), 581-5, 2006.

14. Iwasaki T, Shiba N, Matsuse H, Nago T, Umezu Y, Tagawa Y, Nagata K, Basford JR. Improvement in Knee Extension Strength through Training by Means of Combined Electrical Stimulation and Voluntary Muscle Contraction. Tohoku J. Exp. Med. 209(1), 33-40, 2006.

15. Takano Y, Haneda Y, Maeda T, Sakai Y, Matsuse H, Kawaguchi T, Tagawa Y, Shiba N. Increasing muscle strength and mass of thigh in elderly people with the hybrid-training method of electrical stimulation and volitional contraction. Tohoku J Exp. 221(1):77-85, 2010.

16. Shiba N, Matsuse H, Takano Y, Yoshimitsu K, Omoto M, Hashida R, Tagawa Y, Inada T, Yamada S, Ohshima H.Correction: Electrically Stimulated Antagonist Muscle Contraction Increased Muscle Mass and Bone Mineral Density of One Astronaut - Initial Verification on the International Space Station. PLoS One. 2015 Sep 14;10(9):e0138519.

17. Matsuse H, Shiba N, Takano Y, Yamada S, Ohshima H, Tagawa Y.Cycling exercise to resist electrically stimulated antagonist increases oxygen uptake in males: Pilot study. J Rehabil Res Dev. 50(4).545-554, 2013.

18. Omoto M, Matsuse H, Takano Y, Yamada S, Ohshima H, Tagawa Y, Shiba N. Oxygen Uptake during Aerobic Cycling Exercise Simultaneously Combined with Neuromuscular Electrical

Stimulation of Antagonists. J Nov Physiother. 3-6, 2013. 
19. Aguiar GC, Nascimento MR, De Miranda AS, Rocha NP, Teixeira AL, Scalzo PL.Effects of an exercise therapy protocol on inflammatory markers, perception of pain, and physical performance in individuals with knee osteoarthritis. Rheumatol Int. 2014 Oct 10.

20. Vincent KR, Vincent HK. Resistance exercise for knee osteoarthritis. PMR. 2012 May;4(5 Suppl):S45-52.

21. Lluch Girbés E, Nijs J, Torres-Cueco R, López Cubas C. Pain treatment for patients with osteoarthritis and central sensitization. Phys Ther. 2013 Jun;93(6):842-51.

22. Murphy SL, Lyden AK, Phillips K, Clauw DJ, Williams DA. Subgroups of older adults with osteoarthritis based upon differing comorbid symptom presentations and potential underlying pain mechanisms. Arthritis Res Ther. 2011 Aug 24;13(4):R135.

23. Nijs J, Kosek E, Van Oosterwijck J, Meeus M. Dysfunctional endogenous analgesia during exercise in patients with chronic pain: to exercise or not to exercise? Pain Physician. 2012 Jul;15(3 Suppl):ES205-13

24. Hosseinzadeh M, Andersen OK, Arendt-Nielsen L, Madeleine P. Pain sensitivity is normalized after a repeated bout of eccentric exercise. Eur J Appl Physiol. 2013 Oct;113(10):2595-602.

25. Hosseinzadeh M, Samani A, Andersen OK, Nosaka K, Arendt-Nielsen L, Madeleine P. I psilateral resistance exercise prevents exercise-induced central sensitization in the contralateral limb: a randomized controlled trial. Eur J Appl Physiol. 2015 Nov;115(11):2253-62.

26. Nijs J, Malfliet A, Ickmans K, Baert I, Meeus M.Treatment of central sensitization in patients with 'unexplained' chronic pain: an update. Expert Opin Pharmacother. 2014 Aug;15(12):167183.

27. da Graca-Tarragó M, Deitos A, Patrícia Brietzke A, Torres IL, Cadore Stefani L, Fregni F, Caumo W. Electrical Intramuscular Stimulation in Osteoarthritis Enhances the Inhibitory Systems in Pain Processing at Cortical and Cortical Spinal System. Pain Med. 2015 Sep 23.

28. Gajewska-Woźniak O ${ }^{1}$, Skup M, Kasicki S, Ziemlińska E, Czarkowska-Bauch J. Enhancing proprioceptive input to motoneurons differentially affects expression of neurotrophin 3 and brain-derived neurotrophic factor in rat hoffmann-reflex circuitry. PLoS One. 2013 Jun 11;8(6):e65937. doi: 10.1371/journal.pone.0065937. Print 2013.

29. Yamaguchi T, Tanabe S, Watanabe T, Muraoka Y. Effect of voluntary contraction with electrical stimulation to antagonist muscle on agonist H-reflex. Electromyogr Clin Neurophysiol. 2007 Jul;47(4-5):251-5.

30. Segal NA, Torner JC, Felson D, Niu J, Sharma L, Lewis CE, et al. Effect of thigh strength on incident radiographic and symptomatic knee osteoarthritis in a longitudinal cohort. Arthritis Rheum 2009;61(9):1210-7.

31. 